\title{
Bumblebee sonication behavior changes with plant species and environmental conditions
}

\author{
Callin M. Switzer ${ }^{1}$, Stacey A. COMBes ${ }^{2}$ \\ ${ }^{1}$ Department of Organismic and Evolutionary Biology, Harvard University, 26 Oxford St, Cambridge, MA 02138, USA \\ ${ }^{2}$ Department of Neurobiology, Physiology, and Behavior, University of California, Davis, Davis, CA 95616, USA
}

Received 19 April 2016 - Revised 16 July 2016 - Accepted 9 August 2016

\begin{abstract}
Bumblebees collect pollen from some plants by grasping flowers and vibrating their flight muscles - a behavior termed buzz pollination, or sonication. The extent to which bees can and do alter their sonication has been scarcely documented. We show that bumblebees are capable of changing their vibration frequency. In two field studies, we found that relative humidity, date, and bee size are associated with sonication frequency and/or duration. We found that the frequency and duration of sonications differed on different plants. In a greenhouse study, we found that individual bumblebees change their sonication frequency and duration when collecting pollen from flowers of three different Solanum plants. This suggests that bees may change their sonication behavior to optimize pollen release from different types of flowers.
\end{abstract}

buzz pollination / Solanum / vibration / audio / Bombus impatiens

\section{INTRODUCTION}

Approximately $8 \%$ of flowering plants ( 20,000 species) have poricidal anthers, which release pollen only through small pores (Buchmann 1983). These anthers release pollen when they are vibrated by bees performing buzz pollination, or sonication (Buchmann 1983; King and Buchmann 2003) - a behavior in which bees grasp the anthers with their mandibles (usually) and vibrate their bodies by activating their flight muscles while "decoupling" the wings (King et al. 1996). This transfers the vibration to the flowers, and pollen pours out of the anthers onto the bees' bodies, where it can be groomed and brought to

Electronic supplementary material The online version of this article (doi:10.1007/s13592-016-0467-1) contains supplementary material, which is available to authorized users.

Corresponding author: C. Switzer,

callin.switzer@gmail.com

Manuscript editor: James Nieh the nest to provision the larvae (Buchmann and Cane 1989).

Not all bees perform buzz pollination. Notably, honeybees (Apis mellifera L.) do not sonicate to collect pollen (King and Buchmann 2003), which makes them relatively poor pollinators of plants like tomatoes (Greenleaf and Kremen 2006), peppers (Kwon and Saeed 2003), blueberries (Javorek et al. 2002), and cranberries (OrtwineBoes and Silbernagel 2003). Bumblebees, however, perform sonication on many different flowers and are important commercial pollinators of tomatoes grown in greenhouses (Greenleaf and Kremen 2006).

Some physical characteristics of sonication, including the vibration frequency, amplitude, and duration, affect the rate of pollen release from plants (De Luca et al. 2013; De Luca and Vallejo-Marín 2013). There is evidence that bees can change their sonication behavior, possibly to optimize foraging efficiency. Previous work has shown that two types of buzzes (defensive vs. pollination) are different in terms of frequency, amplitude, and duration in several bumblebee 
species (De Luca et al. 2014). In addition, Morgan et al. (2016) found that Bombus terrestris finetunes its floral sonication behavior-both frequency and amplitude - as it gains experience with handling flowers.

However, because the flight muscles of bees are asynchronous (i.e., a single neural impulse triggers several contractions through stretch activation; Josephson et al. 2000), the extent to which bees are capable of changing their contraction frequency is understudied, though frequency is thought to be limited by physical and physiological properties, rather than being under behavioral control (De Luca and Vallejo-Marín 2013). If a bee's mesosoma operates as a resonant system during buzzing (when the wings do not flap) as well as during flight, several of their sonication characteristics may be correlated - for instance, an increase in buzzing frequency may be associated with a decrease in amplitude. Finally, the duration of sonication may be energetically or thermally constrained (Kammer and Heinrich 1974).

Sonication behavior in bumblebees has been documented multiple times on different types of flowers (Knudsen and Olesen 1993; King and Buchmann 2003; De Luca et al. 2013; Switzer et al. 2016), but the question of whether individual bees alter their sonication behavior on different plants remains unresolved. One study suggested that bees change the length of their sonication bouts in response to pollen output (Kawai and Kudo 2009). Another study demonstrated that two wild bumblebee species (Bombus friseanus Skorikov and Bombus festivus Smith) sonicate at different frequencies on different plants (Corbet and Huang 2014). However, environmental factors and potential differences in body size were not randomized or experimentally manipulated by Corbet and Huang (2014). Furthermore, individual bees were not identified. Thus, the possibility exists that different populations of these wild bees (with different sonication behaviors) were visiting the different types of plants.

We conducted two large field studies (involving $>400$ wild bumblebees, Bombus impatiens Cresson), as well as a greenhouse experiment that allowed for repeated observations on known individuals, to investigate whether bumblebees can and do alter their sonication behavior on different types of plants. We documented environmental conditions (temperature and relative humidity) and body size during field studies, allowing us to account for these additional factors and determine whether they may affect sonication. This variety of approaches allowed us to determine (1) whether bees are capable of changing their sonication frequency (comparing individual bees' irritation buzzes vs. sonication buzzes), (2) whether environmental conditions and body size are associated with how bees sonicate, and (3) whether bees vary their sonication frequency and/or duration when sonicating on different species of plants.

\section{MATERIALS AND METHODS}

\subsection{Field studies on wild Bombus impatiens}

Field investigations were conducted in an urban environment (Arnold Arboretum, Boston MA, $42.29988,-71.12344$ ) and a relatively rural environment (Concord Field Station, Bedford MA, 42.50779, -71.29311). Approximately 40 days of observations were conducted on wild Bombus impatiens between June 14, 2013, and September 17, 2013, typically between the hours of 08:00 and 13:00. We obtained weather information from a weather station at the Arnold Arboretum and from a weather station at Hanscom Air Force Base, near the Concord Field Station.

During each day of observation, we recorded audio clips of bees' sonication buzzes on one or two different species of flower (see Online Resources for flower descriptions and Online Resource Figures 1 and 2 for photos). We analyzed sonication data collected only on plant species for which we recorded at least 20 individuals of B. impatiens .

After recording an individual bee sonicating for pollen collection, we captured the bee with a net. We placed the net on the ground, and while the bee was still in the net, we gently pressed against the bee with the foam tip of the microphone to elicit and record irritation (also called "defense" or "alarm") buzzing - another behavior in which the bee makes an audible buzzing sound without flapping its wings. We excluded irritation buzzes that occurred while the foam on the microphone was touching the bee, because pressure on the bee may 
cause a change in irritation buzz frequency. After recording irritation buzzes, we transferred the bee to a plastic container in a cooler, to narcotize the bee for later measurement and identification. We also recorded wingbeat frequency of flying bumblebees prior to capture, as they approached or flew between flowers; however, we do not report differences between wingbeat frequency and sonication frequency here, because research has already established that sonication frequency is significantly higher than wingbeat frequency in wild B. impatiens (Switzer et al. 2016).

After returning to the lab, we measured each bee's intertegular (IT) span (distance between wing bases) and mass and we marked the bee to avoid performing repeated observations on the same individuals in the field; thus, sonication data was not collected from bees that had previously been captured.

In total, we collected data from 356 wild B. impatiens workers sonicating on five different plants (Callicarpa cathayana Chang, Callicarpa japonica Thunb., Hypericum "Hidcote," Rosa "Bucbi," and Rubus odoratus L.) at the Arnold Arboretum and from 90 workers sonicating on three different plants (Rosa multiflora Thunb., Coronilla varia L., and Physalis philadelphica Lamarck) at the Concord Field Station (see Online Resources for further descriptions of the plants).

\subsection{Greenhouse study on commercial B. impatiens}

We further investigated sonication behavior on different species of plants by performing an experiment with two class-A colonies of $\boldsymbol{B}$. impatiens that were purchased from Biobest (http:// www.biobestgroup.com). Both experimental hives arrived on July 22, 2015. Upon receiving the hives, we verified that queens were present in each, and we removed any males. Each hive was placed in a mesh cage $(1.8 \times 1.8 \times 0.6 \mathrm{~m}$ wide $)$ within an outdoor, pollinator-excluding greenhouse with natural airflow (i.e., exposed to outdoor temperature and humidity).

We allowed bees to acclimate to the cages for at least 2 days prior to marking foragers, and we conducted the experiment over 6 days, from August 13 to 20, 2015.
The hives were enclosed in foam coolers for insulation, with holes for entry and exit. Each cage contained feeders that provided pollen and nectar ad libitum. Nectar consisted of 1.0 M sugar water (organic cane sugar). Pollen was purchased from Koppert Biological Systems (http:// www.koppert.com), and $\sim 2 \mathrm{~g}$ of pollen ground with a mortar and pestle was placed in the feeder approximately every 3 days. In addition to the artificial feeders, each cage contained a potted tomato plant (Solanum lycopersicum "Cherry Roma"). This plant was replaced each day with a different plant that had been kept in a pollinatorexcluding greenhouse, to constantly provide freshly opened flowers for pollen foraging (See Online Resources and Online Resource Figure 3 for descriptions and photos of plants).

Before collecting experimental data, we marked bees that were observed sonicating on $S$. lycopersicum by applying numbered bee tags (Queen Marking Kit, Abelo, Full Sutton, York, UK) to their mesosoma with superglue. The first time a bee was observed foraging on $S$. lycopersicum, we captured her with an insect vacuum (2820GA, BioQuip, Rancho Dominguez, CA) and transferred her from the aspirator tube into a queen-marking cage with plunger (The Bee Works, Oro-Medonte, Ontario, Canada). We gently pressed the bee against the mesh at the top of the tube to immobilize her while we glued a bee tag to her mesosoma. We used the outward vent from the insect vacuum to blow air onto the glue for $30 \mathrm{~s}$ to dry it before releasing the bee back into the cage. We collected experimental data only on bees that had been observed sonicating and were tagged, and then subsequently resumed sonication behavior; this prevented the tagging process from confounding our results (unpubl. data).

After the majority of sonicating bees had been marked with bee tags, we began collecting audio data from marked bees sonicating on different plants. During each audio recording session, we rotated pots of three different Solanum species (Solanaceae) into the bee cages-Solanum lycopersicum L., Solanum dulcamara L., and Solanum carolinense L. (see Online Resources for a description of all plants). The $S$. lycopersicum plants were kept in cages overnight, but we rotated fresh $S$. lycopersicum plants 
into the cages before collecting data. We estimated that the $S$. carolinense plants had fewer flowers than the other two species, but in general the plants had less than 10 open flowers. We rarely recorded bees sonicating on virgin flowers, since all flowers would usually be visited within a few minutes of placing the plant into the cage. We used at least three individual plants of $S$. carolinense and $S$. dulcamara, and we used $\sim 15$ individual plants of $S$. lycopersicum .

We started each session of data collection by placing a potted $S$. lycopersicum plant that had been outside the cage overnight into the cage. After we noticed a decrease in foraging activity on the plant in the cage (median time in cage $\sim 20 \mathrm{~min}$ ), we removed the $S$. lycopersicum plant and replaced it with either $S$. dulcamara or $S$. carolinense, not always in the same order. We again collected recordings until we noticed a decrease in activity ( $20 \mathrm{~min}$ for all plants), and then we replaced the plant in the cage with the species that had not yet been used.

\subsection{Collecting and analyzing audio recordings}

In both field and greenhouse studies, we used a shotgun microphone (SGM-1X, Azden, Tokyo, Japan) to collect audio recordings of sonication with a digital recorder (DR100mkII, Tascam, Montebello, CA). We held the microphone as close to the bee as possible, without touching the bee, attempting to hold the microphone orthogonal to the bees' frontal plane. We were not able to maintain this precise position for all recordings, but we have no evidence that recording from different angles affects the analysis of sonication frequency or duration. When more than one bee was on a flower, we gently brushed one bee off with the foam tip of the microphone, ensuring that each recording contained only one sonicating individual.

We used R (R Core Team 2015), with the packages seewave (Sueur et al. 2008) and tuneR (Ligges et al. 2013), to process the audio recordings and quantify sonication frequency and duration. See Online Resources for further descriptions of processing audio recordings.

\subsection{Statistical analyses}

\subsubsection{Field studies}

We compared irritation buzzes with pollination buzzes by pooling data from the Arnold Arboretum and the Concord Field Station, excluding individuals for which we were unable to process irritation buzz frequency. To evaluate how much individual bees could vary their buzz frequency between pollen collection and irritation buzzing, we used a paired $t$ test to compare sonication frequency and irritation buzz frequency.

To evaluate whether sonication behavior differed on different types of plants, we analyzed field data collected at the Arnold Arboretum and the Concord Field Station separately, because we had no plants that were observed in both locations.

We conducted two stepwise regression procedures for each location - one regression for sonication length and one for sonication frequency. We log-transformed (base e) sonication length to help it fit the assumptions of linear regression. See Online Resources for model selection, evaluation of model assumptions, and multiple comparison adjustments.

\subsubsection{Greenhouse study}

We used a cross-over design for the experiment with colonies of bumblebees foraging in cages within the outdoor greenhouse. In this experiment, bees were allowed to forage on multiple plants, but not every bee foraged on all three of the plant species (S. lycopersicum, S. dulcamara, and $S$. carolinense).

We analyzed bees' sonication frequency and sonication length using two separate multilevel models, with the R package, lme4 (Bates et al. 2015). We log-transformed (base e) sonication length to help it fit the assumptions of linear regression. After finding significant models, we conducted three post hoc tests to compare all pairwise combinations of plants for differences in sonication frequency and sonication length. See Online Resources for model selection, evaluation of assumptions, and multiple comparison adjustments. 


\section{RESULTS}

\subsection{Field studies on wild $B$. impatiens}

We first investigated the question of whether bees can change their sonication frequency by comparing the irritation buzzes of individual bees ( $n=399$ ) with the pollen-collecting buzzes they produced when foraging. Bees recorded at the field sites were capable of producing irritation buzzes that were an average of $90 \mathrm{~Hz}$ higher in frequency than those produced during pollen collection $\left(t_{(398)}=44.47\right.$; two-sided $p$ value $<2.2 \times 10^{-16} ; n=399$; Online Resource Table 1).

At the Concord Field Station, we recorded buzz pollination by 90 wild $B$. impatiens workers on three species of plants (all different from the plants in the Arnold Arboretum), over 13 days of recording. We report models with lowest Bayesian information criterion (BIC). We found that sonication frequency was significantly associated with plant species, while accounting for differences in bees' masses and IT spans $\left(F_{(4,85)}=4.022 ; p\right.$ value $=0.0049$; see Online Resource Table 2 for coefficients and sample sizes). Sonication length was associated with plant species, while accounting for relative humidity $\left(F_{(3,86)}=6.367 ; p\right.$ value $=0.0006$; Online Resource Table 3). Both models had overall $p$ values well below the adjusted significance level of 0.025 . Holding other variables constant, larger masses were associated with higher sonication frequency. Holding other variables constant, larger IT spans were associated with lower sonication frequency at the Concord Field Station (Online Resource Table 2). Higher relative humidity was associated with longer sonication bouts at the Concord Field Station (Online Resource Table 3). Post hoc tests revealed that R. multiflora was sonicated at a significantly higher frequency than were the other plants at the Concord Field Station, after accounting for bee mass and IT span (Figure 1a; Online Resource Table 4); however, we found no significant differences in the length of sonication between these plants after accounting for relative humidity (Figure 1b; Online Resource Table 5).

At the Arnold Arboretum, we recorded buzz pollination by 356 wild $B$. impatiens workers on five species of plants, over 29 days of recording.
These recordings revealed that both sonication frequency and sonication length were significantly associated with plant species, while accounting for differences in relative humidity and date (frequency, $F_{(6,349)}=10.98 ; p$ value $=3.24 \times 10^{-11}$; length, $F_{(7,348)}=7.321 ; p$ value $=3.34 \times 10^{-8}$; see Online Resource Tables 6 and 7 for regression coefficients and sample sizes). These $p$ values are well below the adjusted significance level of $\alpha=0.025$. Both models at the Arnold Arboretum included relative humidity and date. The model for log (base e) sonication length also included date ${ }^{2}$. Higher relative humidity and recordings later in the summer were associated with higher sonication frequencies at the Arnold Arboretum (Online Resource Table 6). For sonication length at the Arnold Arboretum, higher relative humidity was associated with longer sonication bouts and bees tended to perform shorter sonication bouts during the middle of the summer than at the beginning or end (Online Resource Table 7). After accounting for these variables, post hoc tests revealed that bumblebees at the Arnold Arboretum displayed significantly different sonication frequencies and durations on different plants (Figure 1b, d; Online Resource Tables 8 and 9).

\subsection{Greenhouse study on commercial B. impatiens}

We recorded 237 bouts of buzz pollination by $64 \mathrm{~B}$. impatiens workers on three different species of plants over 6 days of recording in the greenhouse. Of the 64 workers, 18 individuals were recorded while sonicating on all three species of plants. Twenty-three individuals were recorded on two different plant species, and 23 were recorded on only one plant species. This study was designed as a cross-over experiment-ideally, every bee in the experiment would have sonicated on all three types of plants, but we were unable to record every bee foraging on each type of plant. However, when we examined only the subset of bees that were recorded on all three plants, we found very similar results as when the entire dataset was used-the regression coefficients were almost identical, but the standard errors were larger for the smaller dataset (Online Resource 


\section{Concord Field Station}

a

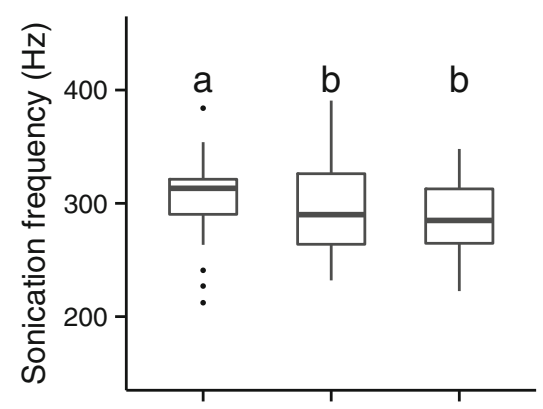

Arnold Arboretum

b

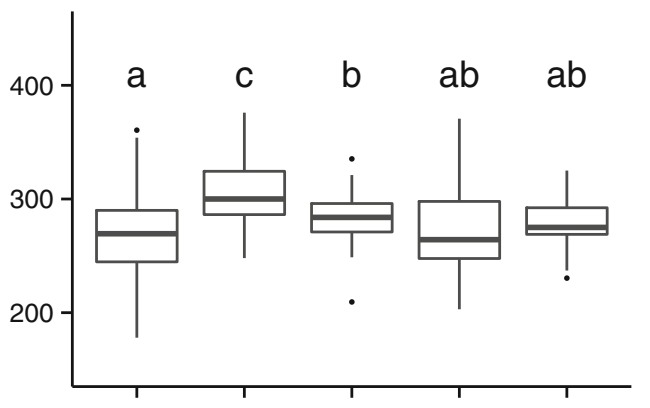

d

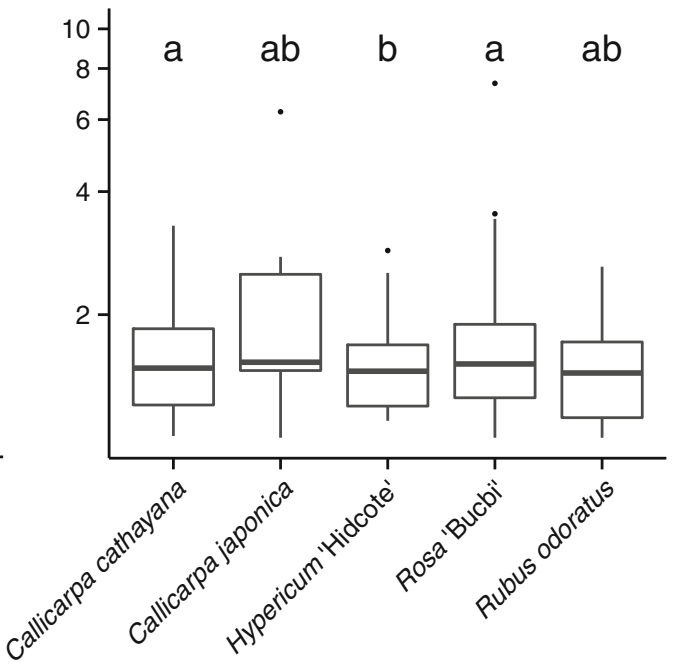

Figure 1. Box plots showing differences in sonication frequency and length at the Concord Field Station (a, c) and at the Arnold Arboretum (b, d ). Different letters above the boxes indicate plants that were sonicated at significantly different frequencies or lengths of time by bumblebees $(p<0.017$ for $\mathbf{a}, \mathbf{c} ; p<0.005$ for $\mathbf{b}$, d ). Note that differences in behaviors were calculated after accounting for associations with other covariates. At the Concord Field Station, differences in frequency (a) account for bee mass and IT span, while differences in length (c) account for relative humidity. At the Arnold Arboretum, differences in frequency (b) and length (d) account for differences in relative humidity and date. Whiskers indicate the highest or lowest values that are within $1.5 *$ interquartile range (IQR), and dots represent individuals outside $1.5 *$ IQR.

Tables 10,11, 12, and 13); thus, the entire dataset was used for further analysis.

Our greenhouse recordings revealed that plant species significantly affected bees' sonication frequency. Based on a likelihood ratio test to compare two multilevel models-one with plant and one without plant as a covariate - we found that plant was a significant predictor of sonication frequency $\left(\chi_{(2)}^{2}=18.788 ; p=8.32 \times 10^{-5} ;\right.$ Online Resource Tables 14 and 15). We used the same process for sonication length and found that plant was also a significant predictor of sonication length, while accounting for the bee 

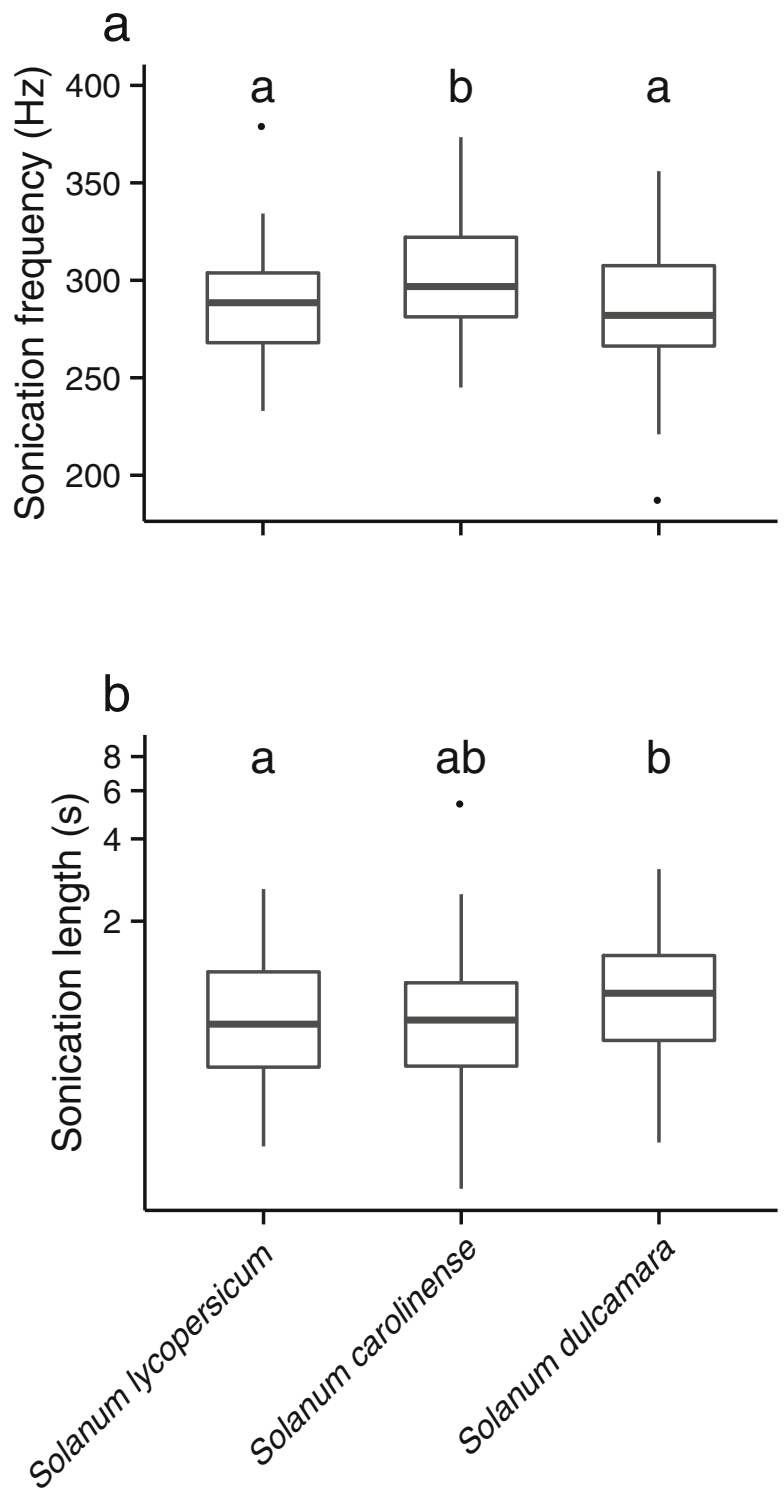

Figure 2. Differences in (a) sonication frequency and (b) sonication length among known Bombus impatiens individuals that were observed sonicating on different Solanum plants in the greenhouse study. Different letters above the boxes indicate plants that were sonicated at significantly different frequencies or over different lengths of time ( $p<0.017$, the adjusted significance level to account for multiple comparisons). Differences in (b) sonication length account for the different bee colonies. Whiskers indicate the highest or lowest values that are within $1.5 *$ interquartile range (IQR), and dots represent observations outside $1.5 *$ IQR.

colony $\left(\chi_{(2)}^{2}=9.216 ; p=0.00997\right.$; Online Resource Tables 16 and 17). Bee colony was a significant predictor for sonication length, but did not make the model for sonication frequency significantly better (according to a likelihood ratio test). Post hoc tests revealed that Solanum carolinense was buzzed at significantly higher frequencies than were the other two plants (Figure 2a; Online Resource Table 18) and that sonication bouts on 
Solanum dulcamara were significantly longer than those on Solanum lycospericum (Figure 2b; Online Resource Table 19).

\section{DISCUSSION/CONCLUSION}

Our paired comparison of irritation vs. pollencollecting buzzes in nearly 400 wild $B$. impatiens workers shows definitively that bumblebees are capable of changing their buzzing frequency. These types of buzzes are superficially similar, in that bees buzz by contracting their flight muscles without flapping their wings; however, bees' highest-frequency irritation buzzes were approximately $90 \mathrm{~Hz}(\sim 30 \%)$ higher than pollencollecting buzzes, demonstrating that bees can buzz at a wide range of frequencies. Our findings support those of De Luca et al. (2014), who found that defense buzzes and pollination buzzes are different. In general, they found that defense buzzes are typically greater in amplitude (louder) than pollination buzzes are. In addition, defense buzzes may not always be higher in frequency compared to pollination buzzes - different bee species show different relationships between buzz type and buzz frequency (De Luca et al. 2014). Results from De Luca et al. (2014) and the current study suggest that the sonication buzzes used while collecting pollen are substantially different from the irritation (also called "alarm," or "defense") buzzes that bees produce in lab settings (see King et al. 1996) and that irritation buzzes may not be a suitable proxy for sonications used to collect pollen.

Furthermore, our studies of wild $B$. impatiens sonicating on different species of plants at the two sites showed that different buzz characteristics are associated with plant species, while accounting for other covariates (discussed below) (Figure 1). While one previous study suggested that sonication characteristics may vary on different plants (Kawai and Kudo 2009), we were able to show this more definitively, by controlling for pseudoreplication of data, accounting for environmental variables and bee size, and collecting data on several hundred bees. The data from the field studies showed that plant species was associated with different sonication frequencies and lengths (Figure 1).
In some cases, differences in flower morphology suggest a potential explanation for the observed differences in buzzing characteristics. For example, flowers of Coronilla varia (also called vetch) are bilaterally symmetric, and we noticed that bees at the Concord Field Station were interacting with this flower differently than with the other flowers. Bees tended to push their heads into the center of C. varia flowers while buzzing, suggesting that they may be using sonication to press their proboscis deeper into the flower to reach the nectar. Clearly, further investigation is necessary to understand the mechanics of this interaction.

In other cases, we could not detect any obvious differences in floral morphology to explain buzz pollination behavior. At the Arnold Arboretum, for example, Callicarpa cathayana was sonicated at a significantly lower frequency than was Callicarpa japonica, although these flowers appear superficially similar; however, other aspects of their biology (amount of pollen produced, number of available flowers, etc.) may underlie the observed differences in sonication characteristics.

Our large dataset of sonication by wild bumblebees in two field sites also allowed us to determine which environmental variables or other covariates may help explain how bees interact with flowers. At the Arnold Arboretum, humidity and date were significant covariates for sonication frequency and sonication length (Online Resource Tables 6 and 7). At the Concord Field Station, the date variable was confounded with plant, and so date was dropped from the analysis. However, relative humidity was also found to be a significant covariate for sonication length at the Concord Field Station (Online Resource Table 3). For sonication frequency measurements at this site, bee mass and IT span were significant covariates (Online Resource Table 2).

Although stepwise procedures are known to sometimes select independent variables that may not actually cause differences in the dependent variables (Burnham and Anderson 2003), our results suggest that relative humidity, bee size, and date may affect sonication characteristics. Our findings on bee size are not surprising, as previous studies have shown that larger bees generally contract their flight muscles at lower frequencies than do small bees, particularly during flight 
(Burkart et al. 2011). This agrees with the coefficient associated with IT span (Online Resource Table 2). Holding bee mass and flower species constant, an increase in IT span was associated with a decrease in sonication frequency at the Concord Field Station (Online Resource Table 2). However, we found the opposite relationship for bee mass - holding IT span and flower species constant, an increase in bee mass was associated with an increase in sonication frequency. This relationship may warrant further investigation in future studies.

Our finding that relative humidity is associated with sonication behavior suggests several interesting hypotheses. Relative humidity may affect sonication behavior through its effects on how much pollen is released-daily cycles of decreasing relative humidity and rising temperature are known to accelerate changes in plant tissue and lead to pollen release (Pacini 2000). In addition, dehydration of tapetal fluid makes pollen easier to remove and high humidity may affect the rate at which the tapetal fluid evaporates (King and Ferguson 1994; King and Buchmann 1996). It is likely that a bee can sense how much pollen gets caught in its pile-flower handling time and grooming time increase when more pollen is available (Buchmann and Cane 1989; Shelly and Villalobos 2000; Burkart et al. 2014). In addition, the properties of pollen grains may change with relative humidity; for example, the volume of pollen grains may increase as relative humidity increases (Gilissen 1977). Future studies investigating the mechanism(s) that allow bees to gauge the amount of pollen landing on their bodies during pollen foraging would be informative. If the weight of the pollen caught in the bee's pile is an indication of the amount of pollen released from the flower, then high humidity may change the bee's behavior because the high-volume pollen grains may be heavier than desiccated pollen grains.

Relative humidity may also affect how well pollen grains attach to bees. Past research has shown that both electrostatic charges and the outer coating on pollen may be important for allowing pollen to attach to pollinators
(Buchmann and Hurley 1978; Vaknin et al. 2000; Pacini and Hesse 2005). A change in relative humidity may affect the electrostatic charge on the bee or the pollen grains, and it may also affect the properties of any coating on the pollen grains. These changes could also affect the size of pollen clumps that are released from the flower. Thus, bees may change their buzz characteristics in response to the amount of pollen sticking to the pile, which may in turn be affected by relative humidity - a hypothesis that warrants future investigation.

Although the results of our field studies clearly showed that different plants were associated with different sonication characteristics, we could not be sure from the field data alone that the plants were actually the cause of the changes in bee behavior. Because we could not observe the same individual on more than one type of plant in the field, the possibility remained that different populations of bees (which sonicated differently) may have been visiting the different plants, rather than individual bees changing their sonication behavior on different plants. To rule this out and to find a causal relationship, we performed the greenhouse study in which known individuals could be observed sonicating on more than one species of plant.

The results of the greenhouse study provided definitive evidence that individual bumblebees alter both their sonication frequency and sonication length on different plants. Bees buzzed at a significantly higher frequency on $S$. carolinense than on the other plants, and buzzes lasted significantly longer on $S$. dulcamara than on S. lycopersicum (Figure 2).

One thing that we cannot determine from this study is whether bees are actively changing their buzzing behavior (e.g., by altering the contraction frequency of their flight muscles) to optimize pollen release or if something about the mechanics of the flower leads to a passive change in the observed buzzing mechanics. The sonication buzzes that bees produce on a flower may be related not only to the bees' muscle contractions and the mechanical properties of its body but also to the resonant properties of the entire system-in other words, mechanical properties of the flower's 
filaments and anthers (mass, stiffness, and damping) could impact the frequency and amplitude of oscillations produced when the bee interacts with it, even if the bee was trying to behave the same way on all of the plants (King and Buchmann 1996). However, passive mechanics would be unlikely to affect how long bees buzz on different types of flowers, which our results show did vary with the type of plant.

We hypothesize that bees change their sonication behavior in response to the pollen rewards they receive. Evidence in the past literature suggests that bees may change their foraging behavior in response to the amount or quality of the reward received from a plant. This has been well studied in nectar foraging. For example, Roubik et al. (1995) suggested that bees forage for optimal nectar concentration, and Manetas and Petropoulou (2000) found that increasing nectar volume causes bees to increase floral visit duration.

Fewer studies have investigated how bees learn and change their foraging behavior when pollen is the reward. Bumblebees have been shown to increase handling time when more pollen is available (Buchmann and Cane 1989), and this could explain why bees sonicated for longer durations on $S$. dulcamara, which may release more pollen than $S$. lycopersicum (unpubl. data). In addition, bumblebees have been shown to "tune" their vibration frequency and amplitude as they gain experience with flowers (Morgan et al. 2016). The current study extends findings from the previous literature concerning pollen foraging behavior and suggests that bees may be able to change their vibration characteristics when foraging for pollen via sonication, in order to optimize pollen collection on different plants (i.e., maximize the amount of pollen collected per buzz).

This study brings up several intriguing questions concerning (1) how the different characteristics of sonication buzzes (e.g., frequency, amplitude, duration) are related and whether these characteristics are constrained to particular ranges; (2) how quickly bees can modify their sonication behavior; and (3) how long their memory of optimal buzzing behavior for different plants persists. Future studies may help shed light on these questions.

\section{ACKNOWLEDGMENTS}

We thank the Arnold Arboretum of Harvard University for providing access to the collections used in this study. We thank Arnold Arboretum collection curator Michael Dosmann and curatorial assistant Kathryn Richardson for their advice and insight. We thank Justin Dower for assisting with data collection and conducting preliminary experiments. We thank Robin Hopkins for detailed reading and suggestions on the manuscript. This material is based upon work supported by the National Defense Science and Engineering Graduate Fellowship (NDSEG) Program to CMS and by the National Science Foundation (CAREER IOS1253677) to SAC.

Le comportement de sonication du bourdon varie en fonction de l'espèce de la plante visitée et des conditions environnementales

Bombus impatiens / Solanum / pollinisation vibratile / vibration / enregistrement sonore

Das Schallbestäubungsverhalten der Hummeln variiert in Abhängigkeit von der Pflanzenart und den Umweltbedingungen

Schallbestäubung / Vibration / Schall / Solanum / Bombus impatiens

\section{REFERENCES}

Bates, D., Mächler, M., Bolker, B. Walker, S. (2015) lme4: Linear mixed-effects models using eigen and S4. https://CRAN.R-project.org/package=lme4 (accessed on 12 Nov 2015)

Buchmann, S.L. (1983) Buzz pollination in angiosperms. in: Jones, C. E. and Little, R. J. (Eds.), Handbook of Experimental Pollination Biology. Van Nostrand Reinhold Company, New York NY, pp. 73-113

Buchmann, S.L., Cane, J.H. (1989) Bees assess pollen returns while sonicating Solanum flowers. Oecologia 81 (3), 289-294

Buchmann, S.L., Hurley, J.P. (1978). A biophysical model for buzz pollination in angiosperms. J. Theor. Biol. 72 (4), 639-657

Burkart, A., Lunau, K., Schlindwein, C. (2011) Comparative bioacoustical studies on flight and buzzing of neotropical bees. J. Pollinat Ecol. 6 (16), 118-124 
Burkart, A., Schlindwein, C., Lunau, K. (2014) Assessment of pollen reward and pollen availability in Solanum stramoniifolium and Solanum paniculatum for buzzpollinating carpenter bees. Plant Biol. 16 (2), 503-507

Burnham, K.P., Anderson, D.R. (2003) Model selection and multimodel inference: a practical informationtheoretic approach. Springer, New York NY

Corbet, S.A., Huang, S.Q. (2014) Buzz pollination in eight bumblebee-pollinated Pedicularis species: does it involve vibration-induced triboelectric charging of pollen grains? Ann. Bot. 114 (8), 1665-1674

De Luca, P.A., Vallejo-Marín, M. (2013). What's the 'buzz' about? The ecology and evolutionary significance of buzz-pollination. Curr. Opin. Plant Biol. 16 (4), 429-435

De Luca, P.A., Bussière, L.F., Souto-Vilaros, D., Goulson, D., Mason, A.C., Vallejo-Marín, M. (2013) Variability in bumblebee pollination buzzes affects the quantity of pollen released from flowers. Oecologia 172 (3), 805816

De Luca, P.A., Cox, D.A., Vallejo-Marín, M. (2014). Comparison of pollination and defensive buzzes in bumblebees indicates species-specific and context-dependent vibrations. Naturwissenschaften 101 (4), 331-338

Gilissen, L.J.W. (1977). The influence of relative humidity on the swelling of pollen grains in vitro. Planta 137 (3), 299-301

Greenleaf, S.S., Kremen, C. (2006) Wild bee species increase tomato production and respond differently to surrounding land use in Northern California. Biol. Conserv. 133 (1), 81-87

Javorek, S.K., Mackenzie, K.E., Vander Kloet, S.P. (2002) Comparative pollination effectiveness among bees (Hymenoptera: Apoidea) on lowbush blueberry (Ericaceae: Vaccinium angustifolium). Ann. Entomol. Soc. Am. 95 (3), 345-351

Josephson, R.K., Malamud, J.G., Stokes, D.R. (2000) Asynchronous muscle: a primer. J. Exp. Biol. 203, 2713-2722

Kammer, A., Heinrich, B. (1974) Metabolic rates related to muscle activity in bumblebees. J. Exp. Biol. 61 (1), 219-227

Kawai, Y., Kudo, G. (2009) Effectiveness of buzz pollination in Pedicularis chamissonis : significance of multiple visits by bumblebees. Ecol. Res. 24 (1), 215-223

King, M.J., Buchmann, S.L. (1996). Sonication dispensing of pollen from Solanum laciniatum flowers. Funct. Ecol. 10 (4), 449-456

King, M.J., Buchmann, S.L. (2003) Floral sonication by bees: mesosomal vibration by Bombus and Xylocopa, but not Apis (Hymenoptera: Apidae), ejects pollen from poricidal anthers. J. Kans. Entomol. Soc. 76 (2), 295-305

King, M.J., Ferguson, A.M. (1994). Vibratory collection of Actinidia deliciosa (kiwifruit) pollen. Ann. Bot. 74 (5), 479-482
King, M.J., Buchmann, S.L., Spangler, H. (1996) Activity of asynchronous flight muscle from two bee families during sonication (buzzing). J. Exp. Biol. 199, 23172321

Knudsen, J.T., Olesen, J.M. (1993) Buzz-pollination and patterns in sexual traits in North European Pyrolaceae. Am. J. Bot. 80 (8), 900-913

Kwon, Y.J., Saeed, S. (2003) Effect of temperature on the foraging activity of Bombus terrestris L. (Hymenoptera: Apidae) on greenhouse hot pepper (Capsicum annuиm L). Appl. Entomol. Zool. 38 (3), 275-280

Ligges, U., Krey, S., Mersmann, O. Schnackenberg, S. (2013) tuneR: Analysis of music. http://r-forge.rproject.org/projects/tuner/ (accessed on 09 Jan 2016)

Manetas, Y., Petropoulou, Y. (2000). Nectar amount, pollinator visit duration and pollination success in the Mediterranean shrub Cistus creticus. Ann. Bot. 86 (4), 815-820.

Morgan, T., Whitehorn, P., Lye, G.C., Vallejo-Marín, M. (2016). Floral sonication is an innate behaviour in bumblebees that can be fine-tuned with experience in manipulating flowers. J. Insect Behav. 29 (2), 233-241

Ortwine-Boes, C. Silbernagel, J. (2003) Bumblebee conservation in and around cranberry marshes, School Proceedings. Wisconsin Cranberry Crop Management Library, pp. 10

Pacini, E. (2000) From anther and pollen ripening to pollen presentation. Plant Syst. Evol. 222 (1), 19-43

Pacini, E., Hesse, M. (2005). Pollenkitt-its composition, forms and functions. Flora 200 (5), 399-415

R Core Team. (2015) R: A language and environment for statistical computing. https://www.r-project.org/ (Accessed on 12 Oct 2015)

Roubik, D.W., Yanega, D., Buchmann, S.L., Inouye, D.W. (1995). On optimal nectar foraging by some tropical bees (Hymenoptera: Apidae). Apidologie 26 (3), 197211

Shelly, T.E., Villalobos, E. (2000) Buzzing bees (Hymenoptera: Apidae, Halictidae) on Solanum (Solanaceae): Floral choice and handling time track pollen availability. Fla Entomol. 83 (2), 180

Sueur, J., Aubin, T., Simonis, C. (2008) Seewave: a free modular tool for sound analysis and synthesis. Bioacoustics 18 (2), 213-226

Switzer, C.M., Hogendoorn, K., Ravi, S., Combes, S.A. (2016) Shakers and head bangers: differences in sonication behavior between Australian Amegilla murrayensis (blue-banded bees) and North American Bombus impatiens (bumblebees). Arthropod-Plant Interact. 10, 1-8

Vaknin, Y., Gan-Mor, S., Bechar, A., Ronen, B., Eisikowitch, D. (2000). The role of electrostatic forces in pollination. Plant Syst. Evol. 222 (1), 133-142 